\title{
Analysis of nurse navigators' activities for hospital discharge coordination: a mixed method study for the case of cancer patients
}

\author{
Fatima Yatim $^{1,2}$ (1) Paula Cristofalo ${ }^{2} \cdot$ Marie Ferrua $^{1} \cdot$ Anne Girault $^{2}$ • \\ Marilene Lacaze $^{1} \cdot$ Mario Di Palma $^{1} \cdot$ Etienne Minvielle $^{1,2}$
}

Received: 21 July 2016/Accepted: 26 October 2016 / Published online: 9 November 2016

(C) The Author(s) 2016. This article is published with open access at Springerlink.com

\begin{abstract}
Background Modern cancer care requires the development of clinical pathways to enhance coordination, but there are few descriptive studies about the content of coordination activities. More specifically, little is known about hospital discharge coordination, although this is seen as a sensitive phase of clinical pathway.

Purpose The purpose of this study was to identify and quantify the categories of activities performed by nurse navigators for hospital discharge coordination.

Methods Patients supported within the Coordinating Outpatient Care department (COC) at Gustave Roussy (Villejuif, France). Study conducted over two consecutive phases (Feb-September 2014): (1) a qualitative phase to identify the categories of coordination activities (interviews with patients plus, focus groups with nurse navigators- $\mathrm{NNs}$ ); (2) a quantitative phase to quantify the relative share of each category. The calls received through the telephone platform of COC (made by both patients and primary care providers) were systematically reported (caller; reason for the call; procedure performed) and then analyzed.

Results Qualitative phase: 17 interviews with patients, plus 2 focus groups with NNs. Quantitative phase: 543 calls analyzed. The callers were patients or their relatives (38\%), private nurses $(35 \%)$, medical device providers $(20 \%)$, and other primary care providers (e.g., pharmacists, family physicians) $(7 \%)$. Five categories of coordination activities
\end{abstract}

Fatima Yatim

fatimayatim15@gmail.com

1 Gustave Roussy, 114, rue Edouard-Vaillant, 94805 Villejuif, France

2 EHESP School Public Health, 15 avenue du Professeur Léon-Bernard, 35043 Rennes, France identified: (F1) Patient monitoring (29\%); (F2) Helping to navigate (24\%); (F3) Managing technical problems (17\%); (F4) Explaining care protocols (16\%); (F5) Collecting and transmitting the patient medical record information (14\%). Conclusions The majority of requirements are related to organizational issues (e.g., navigation, lack of information, appointments). Nurse navigators' training and qualification must therefore combine both clinical and managerial skills.

Keywords Nurse navigator $\cdot$ Discharge coordination . Cancer patients

Thanks to medical advances, cancer becomes a chronic disease. This evolution calls for new healthcare systems organization. Even if other chronic diseases face similar issues, cancer in particular greatly interests healthcare providers and policy-makers because it is responsible for the largest number of deaths, in addition to its social and economic consequences [1-4]. There is a wide consensus therefore that new coordination mechanisms between hospitals and primary care providers-as well as new home patient follow-up methodsare required to introduce a coordinated clinical pathway for cancer patients $[5,6]$. The aim is to fill the gaps relating to fragmentation of the pathway, deficiencies in symptom monitoring, and lack of psychosocial support [7]. To achieve this goal, patient navigation programs (i.e., an intervention that reduces patient barriers to access to care and improves coordination of the clinical pathway) have been implemented [8-11]. Therefore, patient navigators have been increasingly used in an attempt to support patients - either throughout their pathway or during certain sensitive phases [12-15].

However, despite the rising number of experiments being conducted, there is no consensus as to the so-called "coordination activities" of patient navigators [11, 16, 17]. 
The activities described in the literature are various; from patient monitoring [5]; overcoming barriers to accessing the healthcare (e.g., financial, legal, administrative) [10; 13-14]; to patient education and emotional support (e.g., information, advice) $[18,19]$. Furthermore, navigator profiles differ depending on the activities performed [17, 20,21]. Wells et al. [16] show in their literature review that while navigators could be nurses or nurse practitioners, they could also be lay navigators, social workers, health educators or cancer survivors. In their literature review of descriptive and efficacy studies regarding patient navigation programs, Paskett et al. [22] also emphasized the diversity of both patient navigation programs and navigator backgrounds. Moreover, the authors [22] highlighted that the studies had focused on patient navigation programs targeting cancer screening. By comparison, there is little knowledge about patient navigation programs focusing on other phases - such as hospital discharge. Indeed, this phase had been identified as a sensitive one for ensuring patient safety and continuity of care [23-36], and earlier studies highlighted the different unmet patient and primary care providers' needs associated with this phase $[25,26]$.

In fact, little is known concerning the activities devoted to hospital discharge coordination: What are the requests of patients and primary care providers in this phase? What is the content of the activities performed by nurse navigators to respond to these requests? And what are the skills needed? These questions are of interest because findings could impact the recruitment and training of navigators, the implementation of navigation programs, and ultimately could have an impact on patient care efficiency and effectiveness, as well as their satisfaction.

\section{Purpose}

The objective was to identify and quantify the categories of activities performed by nurse navigators for hospital discharge coordination.

\section{Methods}

The case study was conducted at Gustave Roussy Comprehensive Cancer Center (Villejuif, France). Some of the hospitalized patients are physically and mentally autonomous but they require personal assistance due to the complexity of their clinical status (e.g., nutrition problems) or their social conditions (e.g., social isolation), regardless of their pathology. Such patients are followed via the patient navigation program of the Coordinating Outpatient Care (COC) department. The $\mathrm{COC}$ department employs five nurse navigators (NNs) and two assistant nurses in contact with patients and primary care providers, via a telephone platform.
Nurse navigators must have a French official qualification for nurses and a relevant clinical knowledge and skills in oncology. All NNs have proven experience in care coordination and most received a specific training $(80 \mathrm{~h})$ on care coordination, delivered by Paris Sud University and Ecole des Sciences du cancer - Gustave Roussy.

The COC department ensures the follow-up of around 3000 patients per year, and each nurse navigator takes care of 500 patients. Follow-up ends when home care ends, save in exceptional cases.

The main objectives of this patient navigation program are:

1) To organize hospital discharge at the request of inpatient department nurses (coordinating all primary care needed: private nurses, family physicians, pharmacists, etc.)

2) To ensure follow-up at a distance via the telephone platform, from Monday to Friday (ensuring regular outgoing calls (once every 2 weeks), and answering daily incoming calls)

\section{Study design}

The study was conducted over two consecutive phases:

\section{- Qualitative phase}

During this first phase, the main categories of activities performed by NNs, in response to the main requests from patients and primary care providers, were identified. In order to achieve this, focus groups with NNs from the COC department and interviews with patients followed by this department were conducted.

\section{- Quantitative phase}

During this second phase, the calls received through the COC department telephone platform, made by both patients and primary care providers, were systematically reported and analyzed in order to quantify the relative share of each category of NNs' activities.

\section{Qualitative data collection (February-April 2014)}

As a first step, a focus group was conducted with the five NNs. This focus group lasted $2 \mathrm{~h}$ and consisted in describing the content of NNs' daily activities and the main requests from patients and primary care providers.

Patients were subsequently interviewed by two researchers (FY, PC). Prospective patients were identified and approached by NNs to provide a representative sample of patients with regards to age, gender, and specific diagnosis. NNs explained the study to patients and those who agreed were later 
contacted by researchers and interviewed. During the interviews, which lasted on average $2 \mathrm{~h}$, patients were encouraged to speak freely about their experiences with hospital discharge and care coordination as well as about their needs (e.g., difficulties experienced, support provided by NNs).

To complete the qualitative data collection, a second focus group was conducted with the NNs to present and discuss the preliminary results of the first focus group and the interviews with patients.

\section{Qualitative data analysis}

Both focus groups, and all interviews, were audio-recorded for verbatim transcription prepared by a professional transcriptionist, checked for accuracy against the sound files by the interviewers, and corrected where necessary.

The data were analyzed using open coding by two researchers (FY, PC) in an inductive posture [27]. Vertical coding was used in first to identify the activities performed by NNs to meet the requests from patients and primary care providers. Five categories of activities were identified. Vertical coding also made it possible to define the meaning of the items identified within each of these five categories.

Next, horizontal coding was used to combine and compare the meaning of each item. The results generated independently by each researcher were compared and discussed with the principal investigator (EM) until a consensus was reached.

\section{Quantitative data collection (May-September, 2014)}

In order to quantify the relative share of each category of NNs' activities, we developed a quantitative analysis based on a data grid. This was achieved by translating the items defined within the five categories into requests for which patients and primary care providers contact the NNs via the telephone platform of the COC department.

The data grid was refined during an observation period (7 days), and then tested over a period of 2 weeks (10 days), leading to minor adjustments.

Lastly, each call received via the COC telephone platform was systematically reported into the data grid. In addition to reason for the call (chosen among the items proposed; e.g., clinical alert, request for information, or transmission of medical records data), the data grid also included information on call date and caller identity (patient/relative, private nurse, family physician, and others).

\section{Quantitative data analysis}

The data for each grid were entered into a spreadsheet (Excel) and a database was incorporated. Then, the data were summarized using descriptive statistics, to identify the number and percentage of each type of caller and each request for which patients and primary care providers had contacted the COC NNs via the telephone platform. Secondly, pivot tables were used to identify the percentage of each request for each type of caller.

\section{Research ethics}

Participation was entirely voluntary and informed patient consent was obtained systematically. Furthermore, the study was approved by the Gustave Roussy clinical trial department.

\section{Results}

\section{Qualitative results}

In addition to the two focus groups conducted with NNs, 17 interviews with patients were required to achieve empirical data saturation; the additional interviews did not provide any additional information about the five categories of NNs' activities and about the items identified.

The details of patient demographics are shown in Table 1 .

The qualitative data analysis enabled us to identify five categories of NNs' activities, defined as follows:

Patient monitoring (F1)

This category includes requests related to patient monitoring; reporting side effects (e.g., fever, pain), or more rarely reporting an emergency (e.g., discomfort, bleeding, dehydration): "His condition was declining, the patient was not feeling at all well, and the family doctor asked him to return to the hospital, then his wife called us so that we could tell them what to do." (NN-Focus group). Helping to navigate (F2)

Table 1 Patient demographics

\begin{tabular}{llr}
\hline Age & Range (32/78) & \\
(years) & $(14$ of the 17 were & \\
& $\quad$ aged between 45 & \\
and 70 years) & 5 \\
Sex & Female & 12 \\
& Male & 6 \\
Education & Higher level & 11 \\
level & Primary and & 5 \\
& $\quad$ secondary levels & \\
Diagnosis & ENT cancer & 4 \\
& Digestive cancer & 3 \\
& Testicular cancer & 2 \\
& Uterine cancer & 2 \\
& Breast cancer & 1 \\
& Lung cancer & \\
\hline
\end{tabular}


This category includes various requests relating to clinical pathways. A significant portion of calls concerns requests for guidance or simply relaying of contact information (e.g., referring oncologist, dietitian). Reasons could be the need to contact these professionals for advice and/or for appointment-related details (e.g., appointment management, cancelations). However, in some cases, the purpose of the calls can be to alert NNs to, for example, patient's social or psychological difficulties and to ask for assistance. In other cases, the only request is to get a more general explanation regarding pathway organization: "You know, it's a very worrying time. I contacted them just to get further explanations about the pathway and how it works, to reassure me" (Patient 7-Interview). Managing technical problems (F3)

This category includes calls to alert about dysfunctions related to patient care - such as difficulties in drug or medical device delivery or equipment malfunction (e.g., clogged probe, broken pump): "The probe does not work anymore, it is blocked, I tried to call the provider, but they did not respond" (Patient 4-Interview).

Explaining care protocols (F4)

This category includes requests for explanation concerning care protocols - such as a need for clarification about the application of a drug prescription (e.g., frequency, dosage, contraindications), side effects or care techniques (e.g., hydration, infusion duration, use of equipment): "It is not easy, so private nurses prefer to check. They say, you know, I prefer to ask how to do it, so that it goes well." (NN-Focus group).

Collecting and transmitting patient data (F5)

The last category of NNs' activities concerns the sharing and transmission of medical records data (e.g., exam results, hospitalization report): "The patient had been discharged a few days ago, but the doctor had not yet received the hospitalization report, so the private nurse asked me to send it to the doctor" (NN-Focus group). But the vast majority concerns problems relating to prescriptions either missing, illegible, or repeat. In certain cases, the only reason for the call is to obtain or notify diverse information (e.g., announcement of hospitalization).

\section{Quantitative results}

Five hundred forty-three calls received between May and July 2014 via the COC telephone platform were systematically reported and analyzed. The callers were patients or their relatives $(38 \%)$, private nurses $(35 \%)$, medical device providers $(20 \%)$, and other primary care providers (e.g., family physicians, pharmacists) $(7 \%)$.
Requests relating to patient monitoring (F1) account for $29 \%$ of calls; those relating to helping to navigate through the clinical pathway (F2) account for $24 \%$; those relating to managing technical problems (F3) account for $17 \%$; those relating to explaining care protocols (F4) account for $16 \%$; and those relating to collecting and transmitting patient data (F5) account for $14 \%$.

Overall volume of calls for each category of NNs' activities is shown in Table 2:

Call volumes according to the requests from patients and primary care providers are shown in Tables 3 and 4.

Overall, requests relating to clinical monitoring activities (F1) account for $29 \%$ of the calls whereas those relating to what we qualify as organizational activities (F2-F5) account for $71 \%$ of calls.

\section{Discussion}

This case study covers the content of the coordination activities performed by nurse navigators to address the needs of patients and primary care providers after patient discharge.

The first result is the importance of what we qualify as organizational activities during the hospital discharge process. If a significant portion of NNs' activities concern clinical monitoring (F1-29\%) (managing clinical alerts and emergencies), the majority of requirements (71\%) relate to organizational issues: F2 - Helping to navigate through the clinical pathway (24\%); F3-Managing technical problems (17\%); F4-Explaining care protocols (16\%); F5Collecting and transmitting patient data $(14 \%)$. This result offers a comprehensive view of various actions already noticed elsewhere $[17 ; 28-30]$ and corroborates the findings of studies showing that hospital discharge is the handover with the highest level of unmet coordination needs [25, 26]. Indeed, all these activities participate to the effort of coordination during the hospital discharge process.

A second result has to deal with the content of these activities. Our study shows that the main activities performed by NNs involve providing the necessary information (e.g.,

Table 2 NNs' activities according to patients and primary care providers' requests

Volume of calls $(n)$

(F1) Patient monitoring 156

(F2) Helping to navigate through the pathway 130

(F3) Managing technical problems 92

(F4) Explaining care protocols $\quad 87$

(F5) Collecting and transmitting patient data $\quad 76$

Total

543 
Table 3 Patients' (or their relatives) requests

Calls from patients or their relatives

(\%) $(n=211$ phone calls)

(F1) Requests for intervention or clinical advice (side effects or emergencies)

(F2) Requests for guidance, contact information of professionals, or for help to navigate through the pathway

(F3) Requests for intervention to solve various dysfunctions related to patient care

(F4) Requests for explanation concerning care protocols

(F5) Requests for transmission of medical information and data

Total

\section{3}

22

10

16

19

100

announcement of hospitalization, contact information) or intervening directly with various professionals to solve problems or preventing their occurrence through anticipation (e.g., equipment malfunction, social, or psychological difficulties). Their daily activities also include care management well beyond hospital discharge, and general support to patients and primary care providers, to better organize each patient's pathway. All in all, they help articulate the interventions of the various professionals and strengthen the relationship between hospitals and primary care providers, as well as between all these professionals and the patients [31, 32].

Consequently, although such activities necessitate traditional clinical skills, our study suggests that other specific skills are also required. The mobilized skills are in fact not limited to traditional nursing skills relating to care-giving functions [33]; they also include knowledge of the healthcare

Table 4 Primary care providers' requests

\begin{tabular}{ll}
\hline & $\begin{array}{l}\text { Calls from } \\
\text { primary care } \\
\text { providers }(\%) \\
(n=332 \text { phone } \\
\text { calls })\end{array}$ \\
\hline $\begin{array}{l}\text { (F1) Requests for intervention or clinical } \\
\text { advice (side effects or emergencies) }\end{array}$ & 34 \\
$\begin{array}{l}\text { (F2) Requests for guidance, contact information } \\
\text { of professionals, or for help to navigate } \\
\text { through the pathway }\end{array}$ & 11 \\
$\begin{array}{l}\text { (F3) Requests for intervention to solve various } \\
\text { dysfunctions related to patient care }\end{array}$ & 22 \\
(F4) Requests for explanation concerning care \\
protocols
\end{tabular}

system and of available resources within the hospital or the community [28, 34, 35]. This also implies an ability to identify and resolve problems and to understand the different needs of patients and professionals [22, 35]. In addition, this requires some leadership in order to mobilize networks of professionals and create trustworthy relationships between patients and professionals [33]. All these requirements relate to managerial skills, which can be defined as the NNs' ability to play a facilitating role in order to improve the joint performance of all enrolled healthcare providers [36].

With regard to the activities identified for the specific case of hospital discharge, navigators cannot be either lay navigators, social workers, health educators, or cancer survivors. However, nurses could be assisted by other professionalssuch as nursing assistants - for the performance of low value tasks that do not demand clinical or managerial skills (e.g., collecting and transmitting information or medical records data).

\section{Limitations}

The main limitation of this study has to do with the fact that it was conducted in a single cancer center. In addition, the program analyzed herein is available only for autonomous patients but who still require post-discharge support because of the complexity of their clinical or social conditions. The needs of other patients may therefore diverge in some respects. However, although our study findings are specific, they are supported by international studies on patient navigation that have been conducted in other settings. This could be explained by the existence of needs that are common to all patients and healthcare professionals in developed countries, regardless of the situation. Nevertheless, it should be stressed that $G R$ is a French comprehensive cancer center, and then there are certain specificities relating to the French healthcare system, as well as others that concern comprehensive cancer centers exclusively.

\section{Conclusion}

Our findings enrich previous studies on best practices regarding discharge coordination at an organizational level, by offering an on-the-ground analysis of discharge needs from both patients' and primary care providers' perspectives. We have shown that most coordination activities are of organizational nature, thus requiring mainly managerial skills. Training and qualification combining both clinical and managerial aspects are recommended. 
Acknowledgments We thank all patients and the COC department staff at Gustave Roussy.

\section{Compliance with ethical standards}

Conflict of interest The authors declare that they have no conflict of interest.

Open Access This article is distributed under the terms of the Creative Commons Attribution-NonCommercial 4.0 International License (http:// creativecommons.org/licenses/by-nc/4.0/), which permits any noncommercial use, distribution, and reproduction in any medium, provided you give appropriate credit to the original author(s) and the source, provide a link to the Creative Commons license, and indicate if changes were made.

\section{References}

1. Byers T (2010) Two decades of declining cancer mortality: progress with disparity. Annu Rev Public Health 31:121-132

2. Clark CR, Baril N, Kunicki M et al (2009) Addressing social determinants of health to improve access to early breast cancer detection: results of the Boston REACH 2010 Breast and Cervical Cancer Coalition Women's Health Demonstration Project. J Women's Health (Larchmt) 18:677-690

3. Stewart BW, Wild CP (2014) World cancer report

4. Mariotto AB, Yabroff K, Shao Y et al (2011) Projections of the cost of cancer care in the United States: 2010-2020. J Natl Cancer Inst 103(2):117-128

5. Ben-Ami E, Merom H, Sikron F et al (2014) Involvement of the family physician in the care of chemotherapy-treated patients with cancer: patients' perspectives. JOP 10(5):298-305

6. Institute of Medicine (2011) Patient-centered cancer treatment planning: improving the quality of oncology care. Workshop summary. The National Academies Press, Washington, DC

7. Wu C, Bannister W, Schumacker P et al (2014) Economic value of a cancer case management program. JOP 10(3):178-186

8. Freeman HP, Rodriguez RL (2011) History and principles of patient navigation. Cancer 117:3537-3540

9. Freeman HP (2004) A model patient navigator program. Oncol Issues 19:44-46

10. Hede K (2006) Agencies look to patient navigators to reduce cancer care disparities. J Natl Cancer Inst 98:157-159

11. Hendren S, Fiscella K (2014) Patient navigation improves the care experience for patients with newly diagnosed cancer. JCO 1:3-4

12. Rousseau SJ, Humiston SG, Yosha A et al (2014) Patient navigation moderates emotion and information demands of cancer treatment: a qualitative analysis. Support Care Cancer 22:3143-3151

13. Dohan D, Schrag D (2005) Using navigators to improve care of underserved patients: current practices and approaches. Cancer 104: $848-855$

14. Freeman HP (2006) Patient navigation: a community based strategy to reduce cancer disparities. J Urban Health 83:139-141

15. Oluwole SF, Ali AO, Adu A et al (2003) Impact of a cancer screening program on breast cancer stage at diagnosis in a medically underserved urban community. J Am Coll Surg 196:180-188

16. Wells KJ, Battaglia TA, Dudley DJ et al (2008) Patient navigation: state of the art or is it science? Cancer 113:1999-2010
17. Campbell C, Craig J, Eggert J, Bailey-Dorton C (2010) Implementing and measuring the impact of patient navigation at a comprehensive community cancer center. Oncol Nurs Forum 37(1)

18. Basu $\mathrm{M}$ et al (2013) The effect of nurse navigation on timeliness of breast cancer care at an academic comprehensive cancer center. Cancer 119(14):2524-2531

19. Madore S, Kilbourn K, Valverde P et al (2014) Feasibility of a psychosocial and patient navigation intervention to improve access to treatment among underserved breast cancer patients. Support Care Cancer 22(8):2085-2093

20. Shaun L, Wright M, Hodgson S et al (2014) The development and implementation of a volunteer lay navigation competency framework at an outpatient cancer center. Support Care Cancer 22:25712580

21. Lorhan S, Cleghorn L, Fitch M, et al. (2013) Moving the agenda forward for cancer patient navigation: understanding volunteer and peer navigation approaches. J Cancer Educ 1-8

22. Paskett ED, Harrop JP, Wells KJ (2011) Patient navigation: an update on the state of the science. CA Cancer J Clin 61:237-249

23. Kripalani S, LeFevre F, Phillips CO et al (2007) Deficits in communication and information transfer between hospital-based and primary care physicians: implications for patient safety and continuity of care. JAMA 297(8):831-841

24. Jeannie LH, Robert JR, George KF et al (2003) Continuity of care: a multidisciplinary review. BMJ 327:1219

25. Walsh J, Young JM, Harrison JD et al (2011) What is important in cancer care coordination? A qualitative investigation. Eur J Cancer Care 20(2):220-227

26. Søndergaard EG, Grøne BH, Wulff CN et al (2013) A survey of cancer patients' unmet information and coordination needs in handovers - a cross-sectional study. BMC Research Notes 6:378

27. Strauss A, Corbin J (2014) Basics of qualitative research: techniques and procedures for developing grounded theory. Sage Publications

28. Doll R, Stephen J, Barroetavena MC et al (2003) Patient navigation in cancer care: program delivery and research in British Columbia. Can Oncol Nurs J 13:193-193

29. Fillion L, de Serres M, Lapointe-Goupil R et al (2006) Implementing the role of a patient-navigator nurse at a university hospital centre. Can Oncol Nurs J 16:5-17

30. Seek AJ, Hogle WP (2007) Modeling a better way: navigating the healthcare system for patients with lung cancer. Clin J Oncol Nurs 11:81-85

31. Krishnasamy M, Wells M, Wilkie E (2007) Patients and carer experiences of care provision after a diagnosis of lung cancer in Scotland. Supportive Care Cancer 15(3):327-332

32. Jean-Pierre P, Hendren S, Fiscella K et al (2011) Understanding the processes of patient navigation to reduce disparities in cancer care: perspectives of trained navigators from the field. J Cancer Educ 26: $111-120$

33. Allen D (2014) Re-conceptualising holism in the contemporary nursing mandate: from individual to organisational relationships. Soc Sci Med 119:131-138

34. Skrutkowski MSA, Eades M, Swidzinski M et al (2008) Impact of a pivot nurse in oncology on patients with lung or breast cancer: symptom distress, fatigue, quality of life, and use of healthcare resources. Oncol Nurs Forum 35:948-954

35. Wagner EH, Ludman EJ, Bowles EJA et al (2014) Nurse navigators in early cancer care: a randomized, controlled trial. JCO; 32(1):12U85

36. Drucker PF (2006) Classic Drucker: wisdom from Peter Drucker from the pages of Harvard Business Review. Hardcover, March 1 